\title{
Adjacency preservers, symmetric matrices, and cores
}

\author{
Marko Orel
}

Received: 12 September 2010 / Accepted: 13 September 2011 / Published online: 8 October 2011

(C) Springer Science+Business Media, LLC 2011

\begin{abstract}
It is shown that the graph $\Gamma_{n}$ that has the set of all $n \times n$ symmetric matrices over a finite field as the vertex set, with two matrices being adjacent if and only if the rank of their difference equals one, is a core if $n \geq 3$. Eigenvalues of the graph $\Gamma_{n}$ are calculated as well.
\end{abstract}

Keywords Core $\cdot$ Adjacency preserver $\cdot$ Symmetric matrix $\cdot$ Finite field · Eigenvalue of a graph · Coloring · Quadratic form

\section{Introduction}

A graph homomorphism between two undirected graphs $\Gamma$ and $\Gamma^{\prime}$, with no loops and multiple edges, is a map $\Phi: V(\Gamma) \rightarrow V\left(\Gamma^{\prime}\right)$ between the vertex sets such that the following implication holds for any $v, u \in V(\Gamma)$ :

$$
\{v, u\} \text { is an edge } \Longrightarrow\{\Phi(v), \Phi(u)\} \text { is an edge. }
$$

In particular, $\Phi(v) \neq \Phi(u)$ for any edge $\{v, u\}$. A graph homomorphism is an endomorphism if $\Gamma=\Gamma^{\prime}$ and an automorphism if in addition $\Phi$ is bijective, and the converse of (1) also holds (the last statement is redundant if the graph is finite). A graph $\Gamma$ is a core if the semigroup $\operatorname{End}(\Gamma)$ of all its endomorphisms equals the group $\operatorname{Aut}(\Gamma)$ of all its automorphisms. If $\Gamma$ is a graph, then its subgraph $\Gamma^{\prime}$ is called a core of $\Gamma$ if it is a core and there exists some homomorphism $\Phi: \Gamma \rightarrow \Gamma^{\prime}$.

\section{Orel (凶)}

FAMNIT, University of Primorska, Glagoljaška 8, 6000 Koper, Slovenia

e-mail: marko.orel@upr.si 
Let $\mathcal{S}_{n}\left(\mathbb{F}_{q}\right)$ denote the set of all $n \times n$ symmetric matrices over a finite field $\mathbb{F}_{q}$ with $q$ elements. Let $\Gamma_{n}$ be the graph with the vertex set $V\left(\Gamma_{n}\right)=\mathcal{S}_{n}\left(\mathbb{F}_{q}\right)$ and the edge set $E\left(\Gamma_{n}\right)=\{\{A, B\}: \operatorname{rk}(A-B)=1\}$, where rk is the rank of a matrix. It is the main aim of this paper to characterize $\operatorname{End}\left(\Gamma_{n}\right)$ for $n \geq 3$. Since the structure of $\operatorname{Aut}\left(\Gamma_{n}\right)$ is well known [31, Theorem 5.56], it suffices to show that $\Gamma_{n}$ is a core for $n \geq 3$. In the proof we first show that $\Gamma_{n}$ is either a core or it has a complete core. To prove that the latter cannot occur if $n \geq 3$, we show that the chromatic number of $\Gamma_{n}$ is bigger than its clique number. This is done by computing the eigenvalues of $\Gamma_{n}$ and by using Hoffman's lower bound for the chromatic number.

We now state the main results of this paper.

Theorem 1.1 If $n \geq 3$, then $\Gamma_{n}$ is a core. Unless both $q=2$ and $n=3, \operatorname{End}\left(\Gamma_{n}\right)$ consists precisely of the maps of the form

$$
\Phi(A)=a P A^{\sigma} P^{\top}+B
$$

where $a \in \mathbb{F}_{q}$ is a fixed nonzero scalar, $B \in \mathcal{S}_{n}\left(\mathbb{F}_{q}\right), P$ is an invertible $n \times n$ matrix, and $\sigma$ is an automorphism of $\mathbb{F}_{q}$, which is applied entry-wise to $A$. If $q=2$ and $n=3$, then $\operatorname{End}\left(\Gamma_{n}\right)$ is generated by the maps of the form (2) and by the map

$$
\begin{aligned}
\left(\begin{array}{ccc}
a_{11} & a_{12} & a_{13} \\
a_{12} & a_{22} & 0 \\
a_{13} & 0 & a_{33}
\end{array}\right) \mapsto\left(\begin{array}{ccc}
a_{11} & a_{12} & a_{13} \\
a_{12} & a_{22} & 0 \\
a_{13} & 0 & a_{33}
\end{array}\right), \\
\left(\begin{array}{ccc}
a_{11} & a_{12} & a_{13} \\
a_{12} & a_{22} & 1 \\
a_{13} & 1 & a_{33}
\end{array}\right) \mapsto\left(\begin{array}{ccc}
a_{11}+1 & a_{12}+1 & a_{13}+1 \\
a_{12}+1 & a_{22} & 1 \\
a_{13}+1 & 1 & a_{33}
\end{array}\right) .
\end{aligned}
$$

Theorem 1.2 Graph $\Gamma_{2}$ is not a core. Its core is a complete graph on q vertices.

Theorem 1.3 will be used to establish Theorem 1.1, though it is interesting on its own.

Theorem 1.3 Let $n \geq 2$. The (distinct) eigenvalues of $\Gamma_{n}$ are precisely the values $q^{j}-1,-1$, and $-q^{k}-1$, where $j=\left\lceil\frac{n}{2}\right\rceil,\left\lceil\frac{n}{2}\right\rceil+1, \ldots, n$ and $k=\left\lceil\frac{n}{2}\right\rceil,\left\lceil\frac{n}{2}\right\rceil+$ $1, \ldots, n-1$.

The characterization of $\operatorname{Aut}\left(\Gamma_{n}\right)$, where the underlying field is allowed to be infinite, is known as Hua's fundamental theorem of the geometry of symmetric matrices. It was proved in [16] for fields of characteristic not two and in [9, 30] for fields of characteristic two. We also refer to the book [31], which contains the analogous results on hermitian, rectangular, and alternate matrices as well. The fundamental theorem of the geometry of symmetric and hermitian matrices was recently generalized in $[17,18,21]$, where bijective endomorphisms of the corresponding (possibly infinite) graphs were classified. For complex hermitian matrices [20] and real symmetric matrices [23], it was shown that any endomorphism of the corresponding graph is either an automorphism or it has only pairwise adjacent vertices in its image. For a 
graph obtained from $2 \times 2$ symmetric matrices over a field that has odd characteristic and at least seven elements, endomorphisms that have at least two nonadjacent vertices in their image were classified in [19]. A similar result for $2 \times 2$ hermitian matrices over some division rings was obtained in the same paper. The graph on $n \times n$ hermitian matrices over a finite field is a core for any $n$ [26]. The proof relies on the fact that this graph is distance-regular. In [12] it was shown that any non-bipartite distance-regular graph with no triangles is a core.

The rest of this paper is organized as follows. In Sect. 2 we recall definitions and auxiliary theorems that are used in the proofs of the main results. These are contained in Sect. 3. In Sect. 4 several remarks on the main results are listed.

\section{Preliminaries}

We split the preliminaries in two subsections.

\subsection{Finite fields and quadratic forms}

Let $\mathbb{F}_{q}$ denote the finite field with $q$ elements, where $q=p^{k}$ is a power of a prime $p$, and let $\mathbb{F}_{p}:=\{0,1,2, \ldots, p-1\}$ be its prime subfield. Recall that $\mathbb{F}_{p}=\left\{x \in \mathbb{F}_{q}\right.$ : $\left.x^{p}=x\right\}$. The trace map $\operatorname{Tr}: \mathbb{F}_{q} \rightarrow \mathbb{F}_{p}$, defined by $\operatorname{Tr}(x):=x+x^{p}+\cdots+x^{p^{k-1}}$, is surjective (see e.g. [25, Theorem 2.23]), while

$$
\operatorname{Tr}(x)=0 \Longleftrightarrow x=y^{p}-y
$$

for some $y \in \mathbb{F}_{q}\left[25\right.$, Theorem 2.25]. Any $\mathbb{F}_{p}$-linear map $\phi: \mathbb{F}_{q} \rightarrow \mathbb{F}_{p}$ is of the form

$$
\phi(x)=\operatorname{Tr}(y x)
$$

for some $y \in \mathbb{F}_{q}[25$, Theorem 2.24].

A quadratic form in $n$ indeterminates over $\mathbb{F}_{q}$ is a homogeneous polynomial $f \in \mathbb{F}_{q}\left[x_{1}, \ldots, x_{n}\right]$ of degree two or the zero polynomial, that is, $f\left(x_{1}, \ldots, x_{n}\right)=$ $\sum_{i \leq j} c_{i j} x_{i} x_{j}$ for some $c_{i j} \in \mathbb{F}_{q}$. We often denote the column vector $\left(x_{1}, \ldots, x_{n}\right)^{\top}$ by $\mathbf{x}$. We also define $f(\mathbf{x}):=f\left(x_{1}, \ldots, x_{n}\right)$. Two quadratic forms $f, g \in \mathbb{F}_{q}\left[x_{1}\right.$, $\left.\ldots, x_{n}\right]$ are equivalent if there exists an invertible $n \times n$ matrix $P$ with coefficients in $\mathbb{F}_{q}$ such that the forms $f(\mathbf{x})$ and $g(P \mathbf{x})$ are the same. A quadratic form $f\left(x_{1}, \ldots, x_{n}\right)=\sum_{1 \leq i \leq j \leq n} c_{i j} x_{i} x_{j}$ is nondegenerate if it is not equivalent to a form with few indeterminates, i.e., it is not equivalent to a form $\sum_{1 \leq i \leq j \leq n} d_{i j} x_{i} x_{j}$, where $d_{i j}=0$ if $i>i_{0}$ or $j>i_{0}$ for some $i_{0}<n$. We use the symbol $N(f(\mathbf{x})=b)$ to denote the number of column vectors $\mathbf{x} \in \mathbb{F}_{q}^{n}$ such that $f(\mathbf{x})=b$. Clearly, if $f$ and $g$ are two equivalent quadratic forms over $\mathbb{F}_{q}$ in $n$ indeterminates $x_{1}, \ldots, x_{n}$, then $N\left(f\left(x_{1}, \ldots, x_{n}\right)=b\right)=N\left(g\left(x_{1}, \ldots, x_{n}\right)=b\right)$. Moreover, if $f$ is equivalent to a quadratic form $f_{r}$ in $r \leq n$ indeterminates $x_{i_{1}}, \ldots, x_{i_{r}}$, then $N\left(f\left(x_{1}, \ldots, x_{n}\right)=b\right)=$ $q^{n-r} \cdot N\left(f_{r}\left(x_{i_{1}}, \ldots, x_{i_{r}}\right)=b\right)$.

The next lemma follows immediately from [25, Theorem 6.30 and Theorem 6.32]. 
Lemma 2.1 Let $f \in \mathbb{F}_{q}\left[x_{1}, \ldots, x_{n}\right]$ be a nondegenerate quadratic form and $b \in \mathbb{F}_{q}$. If $q$ is even, then the number $N(f(\mathbf{x})=b)$ equals

$$
q^{n-1}
$$

if $n$ is odd, whereas when $n$ is even $N(f(\mathbf{x})=b)$ equals

$$
q^{n-1}+v(b) q^{(n-2) / 2} \text { or } \quad q^{n-1}-v(b) q^{(n-2) / 2} .
$$

Here the map $v: \mathbb{F}_{q} \rightarrow \mathbb{Z}$ is defined by $v(0):=q-1$ and $v(b):=-1$ if $b \neq 0$.

The two possibilities for even $n$ correspond to two different types of quadratic form: hyperbolic type if $N(f(\mathbf{x})=b)=q^{n-1}+v(b) q^{(n-2) / 2}$ and elliptic type if $N(f(\mathbf{x})=$ $b)=q^{n-1}-v(b) q^{(n-2) / 2}$ (see [25] for all the details). If $n=2$, then an example of an elliptic form is given by $x_{1}^{2}+x_{1} x_{2}+a x_{2}^{2}$, where $\operatorname{Tr}(a)=1$ [25, Theorem 6.32]. Therefore,

$$
N\left(x_{1}^{2}+x_{1} x_{2}+a x_{2}^{2}=0\right)=1
$$

and (6) has only trivial solution $x_{1}=0=x_{2}$.

If $q$ is odd, then the form $f\left(x_{1}, \ldots, x_{n}\right)=\sum_{i \leq j} c_{i j} x_{i} x_{j}$ can be written as $f(\mathbf{x})=$ $\mathbf{x}^{\top} C \mathbf{x}$, where $C$ is the $n \times n$ symmetric matrix whose $(i, i)$-entry is $c_{i i}$ and $(i, j)$ entry is $c_{i j} / 2$. In odd characteristic we will rely on the determinant of a quadratic form, which is defined by $\operatorname{det}(f):=\operatorname{det}(C)$. All nondegenerate quadratic forms satisfy $\operatorname{det}(f) \neq 0$. Moreover, if two quadratic forms $f, g \in \mathbb{F}_{q}\left[x_{1}, \ldots, x_{n}\right]$ are equivalent, then $\operatorname{det}(f)=\operatorname{det}(g) \operatorname{det}(P)^{2}$ for some invertible $P$. In the sequel we are concerned only whether a determinant of a given quadratic form is a square or not, so equivalent forms have the 'same' determinant in this sense.

The next lemma is proved in [25, Theorem 6.26 and Theorem 6.27].

Lemma 2.2 Let $f \in \mathbb{F}_{q}\left[x_{1}, \ldots, x_{n}\right]$ be a nondegenerate quadratic form and $b \in \mathbb{F}_{q}$. If $q$ is odd, then

$$
N(f(\mathbf{x})=b)= \begin{cases}q^{n-1}+v(b) q^{(n-2) / 2} \eta\left((-1)^{n / 2} \operatorname{det}(f)\right) & \text { if } n \text { is even } \\ q^{n-1}+q^{(n-1) / 2} \eta\left((-1)^{(n-1) / 2} b \operatorname{det}(f)\right) & \text { if } n \text { is odd }\end{cases}
$$

Here the map $v: \mathbb{F}_{q} \rightarrow \mathbb{Z}$ is defined as in Lemma 2.1, while $\eta: \mathbb{F}_{q} \rightarrow \mathbb{Z}$ is defined by $\eta(0):=0, \eta(c):=1$ if $c$ is a nonzero square, and $\eta(c)=-1$ otherwise.

\subsection{Graphs and symmetric matrices}

From now on all graphs are finite, undirected, and without loops and multiple edges. Given a graph $\Gamma$, we use $\chi(\Gamma)$ to denote its chromatic number, that is, the smallest integer $m$ for which there exists a vertex $m$-coloring, i.e., a map $\mathcal{C}: V(\Gamma) \rightarrow$ $\left\{x_{1}, x_{2}, \ldots, x_{m}\right\}$ on the vertex set, which maps into a set of cardinality $m$ and satisfies $\mathcal{C}(v) \neq \mathcal{C}(u)$ whenever $\{v, u\}$ is an edge. The next simple lemma is well known (see e.g. [22, Proposition 1.20] or [14, Corollary 1.8]). It just observes that a composition of a graph homomorphism and a vertex coloring is still a vertex coloring. 
Lemma 2.3 If $\Phi: \Gamma \rightarrow \Gamma^{\prime}$ is a graph homomorphism, then $\chi(\Gamma) \leq \chi\left(\Gamma^{\prime}\right)$.

In the proof of the main result we rely on Theorem 2.4 [15], which gives a lower bound for the chromatic number in terms of the maximal eigenvalue $\lambda_{\max }$ and the minimal eigenvalue $\lambda_{\min }$ of a graph.

Theorem 2.4 If $\Gamma$ has at least one edge, then $\chi(\Gamma) \geq 1+\frac{\lambda_{\max }}{-\lambda_{\min }}$.

Let $(G,+)$ be a finite abelian group and suppose that a subset $S \subseteq G \backslash\{0\}$ satisfies $-S=S$. The Cayley graph $\operatorname{Cay}(G, S)$ is the graph $\Gamma$ with the vertex set $V(\Gamma)=G$ and the edge set $E(\Gamma)=\{\{g, h\}: g-h \in S\}$. A map $\xi: G \rightarrow \mathbb{C} \backslash\{0\}$ is a character if $\xi(g+h)=\xi(g) \xi(h)$ for all $g, h \in G$. A complex number $\lambda$ is an eigenvalue of $\operatorname{Cay}(G, S)$ if and only if

$$
\lambda=\sum_{s \in S} \xi(s)
$$

for some character $\xi$ (see e.g. [10, Lemma 9.2, p. 246] or [2, Sect. 1.4.8]). If $G=$ $\mathcal{S}_{n}\left(\mathbb{F}_{q}\right)$ is the abelian group of all $n \times n$ symmetric matrices over a finite field and the subset $S \subseteq G$ consists of all matrices of rank one, then $\operatorname{Cay}(G, S)=\Gamma_{n}$. The graph $\Gamma_{n}$ is regular of valency $|S|=q^{n}-1$, but it is not distance-regular unless $n \in\{2,3\}$ and $q$ is even (see [3, pp. 285-286] and [4]). The diameter of $\Gamma_{n}$ equals $n$ if $n$ or $q$ is odd, whereas the diameter equals $n+1$ if both $n$ and $q$ are even [31, Proposition 5.55]. We also mention that the distance $d(A, B)$ between two matrices $A$ and $B$ equals $\operatorname{rk}(A-$ $B$ ) if $q$ is odd. However, if $q$ is even, then $d(A, B)=\operatorname{rk}(A-B)+1$ if $A-B$ is an nonzero alternate matrix and $d(A, B)=\operatorname{rk}(A-B)$ otherwise [31, Proposition 5.5]. Any rank-one symmetric matrix can be written as $a \mathbf{x x}^{\top}$ for some column vector $\mathbf{x}$ and some scalar $a$. If $q$ is even, then any scalar is a square, so we can assume that $a=1$. Let $l$ be a maximum clique in $\Gamma_{n}$, that is, some set of pairwise adjacent matrices, which is of the largest possible size. Then

$$
l=\left\{a \mathbf{x} \mathbf{x}^{\top}+A: a \in \mathbb{F}_{q}\right\}
$$

for some nonzero column vector $\mathbf{x}$ and some $A \in \mathcal{S}_{n}\left(\mathbb{F}_{q}\right)$ [31, Proposition 5.8]. In particular we see that the cardinality $|l|$ equals $q$.

The next theorem is a special case of the fundamental theorem of the geometry of symmetric matrices (see [9, 16, 30] or the book [31, Theorem 5.56]) for matrices over a finite field.

Theorem 2.5 Suppose that $n \geq 2$. Elements of $\operatorname{Aut}\left(\Gamma_{n}\right)$ are precisely the maps of the form (2), unless $q=2$ and $n=3$. In this special case Aut $\left(\Gamma_{n}\right)$ has an additional generator given by the map (3).

\section{Proofs}

We first prove that the graph $\Gamma_{n}$ is either a core or it has a complete core. It was observed by Godsil and Royle [12] that such a property is possessed by many graphs 
with high degree of transitivity on pairs of vertices at distance two. Although their results cannot be applied directly to $\Gamma_{n}$, the following lemma allows us to modify the proof of [12, Theorem 4.1] suitably.

Lemma 3.1 Suppose that $A, B \in V\left(\Gamma_{n}\right)=\mathcal{S}_{n}\left(\mathbb{F}_{q}\right)$ are such that $d(A, B)=2$. Let $l$ be an arbitrary maximum clique in $\Gamma_{n}$ and let $C \in V\left(\Gamma_{n}\right) \backslash l$ be adjacent to some matrix in $l$. Then there exist a matrix $D \in l$ and an automorphism $\Psi$ of $\Gamma_{n}$ such that $\Psi(C)=A$ and $\Psi(D)=B$.

Proof By (8), there exist a column vector $\mathbf{x}$ and a symmetric matrix $X_{0}$ such that $l=\left\{a \mathbf{x} \mathbf{x}^{\top}+X_{0}: a \in \mathbb{F}_{q}\right\}$. Since $C$ is adjacent to some matrix in $l$, there exist scalars $a_{0}, b_{0}$, where $b_{0} \neq 0$, and a column vector $\mathbf{y}$ such that $C=a_{0} \mathbf{x x}^{\top}+X_{0}+b_{0} \mathbf{y y}^{\top}$. The vectors $\mathbf{x}$ and $\mathbf{y}$ must be linearly independent, since $C \notin l$. Hence, there exists an invertible matrix $P$ with $\mathbf{x}$ as the first column and $\mathbf{y}$ as the second column. Consequently, if $E_{j j}$ denotes the matrix with 1 as the $(j, j)$-entry and zeros elsewhere, then $l=\left\{a P E_{11} P^{\top}+X_{0}: a \in \mathbb{F}_{q}\right\}$ and $C=P\left(a_{0} E_{11}+b_{0} E_{22}\right) P^{\top}+X_{0}$. Since $d(A, B)=2$, the difference $A-B$ is a sum of two linearly independent rank-one symmetric matrices. That is, there exist an invertible $Q$ and nonzero $a_{0}^{\prime}$ and $b_{0}^{\prime}$ such that $A-B=Q\left(a_{0}^{\prime} E_{11}+b_{0}^{\prime} E_{22}\right) Q^{\top}$. It is now easy to see that the matrix $D:=\left(a_{0}-b_{0} a_{0}^{\prime} / b_{0}^{\prime}\right) P E_{11} P^{\top}+X_{0}$ and the automorphism $\Psi(X):=$ $\left(b_{0}^{\prime} / b_{0}\right)\left(Q P^{-1}\right)(X-D)\left(Q P^{-1}\right)^{\top}+B$ have the required properties.

In the proof of Lemma 3.2 we tacitly rely on the fact that any graph has a core, which is always an induced graph and unique up to isomorphism [11, Lemma 6.2.2].

Lemma 3.2 Graph $\Gamma_{n}$ is either a core or it has a complete core.

Proof Denote any subgraph of $\Gamma_{n}$ that is a core of $\Gamma_{n}$ by $\Gamma^{\prime}$. For any graph homomorphism $\Phi: \Gamma_{n} \rightarrow \Gamma^{\prime}$, denote the composition $\left(\left.\Phi\right|_{\Gamma^{\prime}}\right)^{-1} \circ \Phi$ by $\Phi^{\prime}$. Then, $\Phi^{\prime}: \Gamma_{n} \rightarrow \Gamma^{\prime}$ is a graph homomorphism that fixes the vertex set $V\left(\Gamma^{\prime}\right)$. Suppose that $\Gamma^{\prime} \neq \Gamma_{n}$, i.e., $V\left(\Gamma^{\prime}\right) \subsetneq V\left(\Gamma_{n}\right)$. Since $\Gamma_{n}$ is connected, there exist $X \in V\left(\Gamma^{\prime}\right)$ and $A \in V\left(\Gamma_{n}\right) \backslash V\left(\Gamma^{\prime}\right)$, which are adjacent in $\Gamma_{n}$. Hence, $B:=\Phi^{\prime}(A)$ is adjacent to $\Phi^{\prime}(X)=X$. Moreover, $\Phi^{\prime}(A)=\Phi^{\prime}(B)$ and $A \neq B$, since $A \notin V\left(\Gamma^{\prime}\right)=\Phi^{\prime}\left(V\left(\Gamma_{n}\right)\right)$. Hence, $d(A, B)=2$. Let $l$ be an arbitrary maximum clique of $\Gamma_{n}$, which is contained in $\Gamma^{\prime}$. Suppose that $\Gamma^{\prime}$ contains two vertices at distance two. Since $\Gamma^{\prime}$ is connected, there exist a matrix $C \in V\left(\Gamma^{\prime}\right) \backslash l$ that is adjacent to some matrix from $l$. By Lemma 3.1, there exist a matrix $D \in l$ and an automorphism $\Psi$ of $\Gamma_{n}$ such that $\Psi(C)=A$ and $\Psi(D)=B$. Consequently, $\Phi^{\prime}(\Psi(C))=\Phi^{\prime}(\Psi(D))$, so the restriction of composition $\Phi^{\prime} \circ \Psi$ to $V\left(\Gamma^{\prime}\right)$ is an element of $\operatorname{End}\left(\Gamma^{\prime}\right) \backslash \operatorname{Aut}\left(\Gamma^{\prime}\right)$. As $\Gamma^{\prime}$ is a core, we get a contradiction. Hence, all vertices in $\Gamma^{\prime}$ are adjacent.

The following lemma is the main step toward the proofs of Theorem 1.1 and Theorem 1.3. A map $\phi: \mathcal{S}_{n}\left(\mathbb{F}_{q}\right) \rightarrow \mathbb{F}_{p}$ is called additive if $\phi(A+B)=\phi(A)+\phi(B)$ for all $A, B \in \mathcal{S}_{n}\left(\mathbb{F}_{q}\right)$.

Lemma 3.3 Let $q$ be a power of a prime $p$ and suppose that $n \geq 2$ is an integer. If $\Delta$ is the set of rank-one matrices in $\mathcal{S}_{n}\left(\mathbb{F}_{q}\right)$ that are annihilated by an additive map 
$\phi: \mathcal{S}_{n}\left(\mathbb{F}_{q}\right) \rightarrow \mathbb{F}_{p}$, then

$$
|\Delta| \in\left\{q^{n}-1, \frac{q^{n}}{p}-1, \frac{q^{n}}{p}-1+q^{n-r / 2}\left(1-\frac{1}{p}\right), \frac{q^{n}}{p}-1-q^{n-r / 2}\left(1-\frac{1}{p}\right)\right\},
$$

where $2 \leq r \leq n$ is an even integer. Moreover, any value in the set (9) is attained by some $\Delta$.

Proof Since $\phi$ is additive, there exist additive maps $\phi_{i j}: \mathbb{F}_{q} \rightarrow \mathbb{F}_{p}$ such that $\phi\left(\left[a_{i j}\right]\right)=\sum_{1 \leq i \leq j \leq n} \phi_{i j}\left(a_{i j}\right)$. Maps $\phi_{i j}$ are in fact linear over the subfield $\mathbb{F}_{p}$, so by (5) there exist scalars $c_{i j} \in \mathbb{F}_{q}$ such that $\phi_{i j}\left(a_{i j}\right)=\operatorname{Tr}\left(c_{i j} a_{i j}\right)$. Since the trace map is additive as well, we see that

$$
\phi=\operatorname{Tr} \circ \psi, \quad \text { where } \quad \psi\left(\left[a_{i j}\right]\right)=\sum_{i \leq j} c_{i j} a_{i j} .
$$

If all $c_{i j}$ are zero, then $\phi \equiv 0$, and $|\Delta|=q^{n}-1$ equals the number of all rankone matrices in $\mathcal{S}_{n}\left(\mathbb{F}_{q}\right)$. Assume now that some $c_{i j}$ is nonzero. Then, there exists $r \in\{1,2, \ldots, n\}$ such that the quadratic form

$$
f(\mathbf{x})=\sum_{i \leq j} c_{i j} x_{i} x_{j}
$$

is equivalent to some quadratic form $f_{r}$ in $r$ indeterminates $x_{i_{1}}, \ldots, x_{i_{r}}$, which is nondegenerate if regarded as a quadratic form in $\mathbb{F}_{q}\left[x_{i_{1}}, \ldots, x_{i_{r}}\right]$. Recall that

$$
N\left(f\left(x_{1}, \ldots, x_{n}\right)=b\right)=q^{n-r} \cdot N\left(f_{r}\left(x_{i_{1}}, \ldots, x_{i_{r}}\right)=b\right)
$$

for any $b$.

To proceed, consider the map $x \mapsto x^{p}-x$ on $\mathbb{F}_{q}$. It is $p$-to-1, since in a field of characteristic $p$ the equivalence

$$
x^{p}-x=y^{p}-y \Longleftrightarrow(x-y)^{p}=x-y \quad \Longleftrightarrow \quad x-y \in \mathbb{F}_{p}
$$

holds. Hence, the map $x \mapsto x^{p}-x$ attains $q / p$ distinct values $d_{1}, \ldots, d_{q / p}$, where one of them, say $d_{q / p}$, is zero. From (4) it follows that

$$
\operatorname{Tr}(x)=0 \Longleftrightarrow x \in\left\{d_{1}, \ldots, d_{q / p}\right\}
$$

for all $x \in \mathbb{F}_{q}$. To end the proof we separate two cases.

Case 1. Let $q$ be even. Then for each $i \in\{1, \ldots, q / p\}$ define

$$
\Delta_{i}:=\left\{\mathbf{x x}^{\top}: \psi\left(\mathbf{x x}^{\top}\right)=d_{i}\right\}
$$

Set $\Delta_{i}$ contains matrices of rank one if $i \neq q / p$, and in addition $\Delta_{i}$ contains the zero matrix if $i=q / p$. Moreover, in a finite field of characteristic $p=2$ any rank-one symmetric matrix is of the form $\mathbf{x} \mathbf{x}^{\top}$. Hence, we can infer from (10) and (13) that the 
Table 1

\begin{tabular}{|c|c|}
\hline \multicolumn{2}{|l|}{$r$ is odd } \\
\hline$\left|\Delta_{i}\right|, i \neq q / p$ & $q^{n-1}$ \\
\hline$\left|\Delta_{q / p} \backslash\{0\}\right|$ & $q^{n-1}-1$ \\
\hline \multicolumn{2}{|c|}{$r$ is even, $f_{r}$ is of hyperbolic type } \\
\hline$\left|\Delta_{i}\right|, i \neq q / p$ & $q^{n-1}-q^{n-1-\frac{r}{2}}$ \\
\hline$\left|\Delta_{q / p} \backslash\{0\}\right|$ & $q^{n-1}+(q-1) q^{n-1-\frac{r}{2}}-1$ \\
\hline \multicolumn{2}{|c|}{$r$ is even, $f_{r}$ is of elliptic type } \\
\hline$\left|\Delta_{i}\right|, i \neq q / p$ & $q^{n-1}+q^{n-1-\frac{r}{2}}$ \\
\hline$\left|\Delta_{q / p} \backslash\{0\}\right|$ & $q^{n-1}-(q-1) q^{n-1-\frac{r}{2}}-1$ \\
\hline
\end{tabular}

matrices in the sets $\Delta_{i}$ are precisely those matrices of rank $\leq 1$ that are annihilated by $\phi$. Since the characteristic equals 2 , we also see that

$$
\mathbf{x}_{1}=\mathbf{x}_{2} \Longleftrightarrow \mathbf{x}_{1} \mathbf{x}_{1}^{\top}=\mathbf{x}_{2} \mathbf{x}_{2}^{\top},
$$

so $\left|\Delta_{i}\right|=N\left(f(\mathbf{x})=d_{i}\right)$. We can now use (12) and Lemma 2.1 to obtain the data in Table 1 . By definition (14), $\Delta_{i} \cap \Delta_{j}=\emptyset$ for $i \neq j$, so

$$
\begin{aligned}
|\Delta| & =\sum_{i=1}^{q / p-1}\left|\Delta_{i}\right|+\left|\Delta_{q / p} \backslash\{0\}\right|=(q / p-1)\left|\Delta_{1}\right|+\left|\Delta_{q / p} \backslash\{0\}\right| \\
& \in\left\{\frac{q^{n}}{p}-1, \frac{q^{n}}{p}-1+q^{n-r / 2}\left(1-\frac{1}{p}\right), \frac{q^{n}}{p}-1-q^{n-r / 2}\left(1-\frac{1}{p}\right)\right\} .
\end{aligned}
$$

Clearly, all values can be attained for a suitable $f$, i.e., for suitable scalars $c_{i j}$.

Case 2. Now let $q$ be odd. Set

$$
b_{r}:= \begin{cases}(-1)^{(r-1) / 2} \operatorname{det}\left(f_{r}\right) & \text { if } r \text { is odd, } \\ 1 & \text { if } r \text { is even, }\end{cases}
$$

and pick an arbitrary non-square $c \in \mathbb{F}_{q}$. From (12) and Lemma 2.2 we obtain the data in Table 2. Denote the (nonzero) column vectors that satisfy $f(\mathbf{x})=b_{r}$ by

$$
\mathbf{x}_{1}, \ldots, \mathbf{x}_{\alpha},-\mathbf{x}_{1}, \ldots,-\mathbf{x}_{\alpha}
$$

the (nonzero) column vectors that satisfy $f(\mathbf{y})=b_{r} c$ by

$$
\mathbf{y}_{1}, \ldots, \mathbf{y}_{\beta},-\mathbf{y}_{1}, \ldots,-\mathbf{y}_{\beta},
$$

and some maximal selection of pairwise linear independent column vectors that satisfy $f(\mathbf{z})=0$ by

$$
\mathbf{z}_{1}, \ldots, \mathbf{z}_{\gamma}
$$

Note that

$$
\alpha=N\left(f(\mathbf{x})=b_{r}\right) / 2
$$


Table 2

\begin{tabular}{ll}
\hline$r$ is odd & \\
$N\left(f(\mathbf{x})=b_{r}\right)$ & $q^{n-1}+q^{n-1-\frac{r-1}{2}}$ \\
$N\left(f(\mathbf{x})=b_{r} c\right)$ & $q^{n-1}-q^{n-1-\frac{r-1}{2}}$ \\
$N(f(\mathbf{x})=0)$ & $q^{n-1}$ \\
\hline$r$ is even, $(-1)^{r / 2} \operatorname{det}\left(f_{r}\right)$ is a square & \\
$N\left(f(\mathbf{x})=b_{r}\right)$ & $q^{n-1}-q^{n-1-\frac{r}{2}}$ \\
$N\left(f(\mathbf{x})=b_{r} c\right)$ & $q^{n-1}-q^{n-1-\frac{r}{2}}$ \\
$N(f(\mathbf{x})=0)$ & $q^{n-1}+(q-1) q^{n-1-\frac{r}{2}}$ \\
\hline$r$ is even, $(-1)^{r / 2} \operatorname{det}\left(f_{r}\right)$ is not a square & \\
$N\left(f(\mathbf{x})=b_{r}\right)$ & $q^{n-1}+q^{n-1-\frac{r}{2}}$ \\
$N\left(f(\mathbf{x})=b_{r} c\right)$ & $q^{n-1}+q^{n-1-\frac{r}{2}}$ \\
$N(f(\mathbf{x})=0)$ & $q^{n-1}-(q-1) q^{n-1-\frac{r}{2}}$ \\
\hline
\end{tabular}

$$
\begin{aligned}
& \beta=N\left(f(\mathbf{y})=b_{r} c\right) / 2, \\
& \gamma=(N(f(\mathbf{z})=0)-1) /(q-1) .
\end{aligned}
$$

In fact, to compute $\gamma$, that is, to achieve pairwise linear independence, we need to exclude the zero column vector and consider only one multiple of any $\mathbf{z}$.

Now, for $i \in\{1, \ldots, q / p-1\}$ define

$$
\begin{aligned}
\Delta_{i, \mathbf{x}} & :=\left\{\left(d_{i} b_{r}^{-1}\right) \cdot \mathbf{x}_{j} \mathbf{x}_{j}^{\top}: j \in\{1, \ldots, \alpha\}\right\}, \\
\Delta_{i, \mathbf{y}} & :=\left\{\left(d_{i} b_{r}^{-1} c^{-1}\right) \cdot \mathbf{y}_{j} \mathbf{y}_{j}^{\top}: j \in\{1, \ldots, \beta\}\right\}, \\
\Delta_{\mathbf{z}} & :=\left\{a \cdot \mathbf{z}_{j} \mathbf{z}_{j}^{\top}: j \in\{1, \ldots, \gamma\}, 0 \neq a \in \mathbb{F}_{q}\right\} .
\end{aligned}
$$

All matrices in the sets $\Delta_{i, \mathbf{x}}, \Delta_{i, \mathbf{y}}, \Delta_{\mathbf{z}}$ are of rank one. Moreover, from the definitions of the vectors $\mathbf{x}_{j}, \mathbf{y}_{j}, \mathbf{z}_{j}$ and from (10), (11), and (13), it follows that any such matrix is annihilated by $\phi$. We claim that these are in fact the only rank-one matrices that are annihilated by $\phi$. Namely, let $\phi\left(a \mathbf{w} \mathbf{w}^{\top}\right)=0$ for some nonzero $a$ and $\mathbf{w}$. Then $\psi\left(a \mathbf{w} \mathbf{w}^{\top}\right)$ equals $d_{i}$ for some $i$. If $\psi\left(a \mathbf{w} \mathbf{w}^{\top}\right)=d_{q / p}=0$, then

$$
0=\psi\left(\mathbf{w} \mathbf{w}^{\top}\right)=f(\mathbf{w}),
$$

so $\mathbf{w}$ is a multiple of some column vector in (15), that is, $a \mathbf{w} \mathbf{w}^{\top} \in \Delta_{\mathbf{z}}$. Assume now that $\psi\left(a \mathbf{w} \mathbf{w}^{\top}\right)=d_{i} \neq 0$. Since $q$ is odd, precisely half of the elements of $\mathbb{F}_{q} \backslash\{0\}$ are squares. Moreover, $\mathbb{F}_{q}=\left\{0, t_{1}^{2}, \ldots, t_{(q-1) / 2}^{2}, c t_{1}^{2}, \ldots, c t_{(q-1) / 2}^{2}\right\}$ for suitable $t_{i}$, so one of the two scalars $b_{r} a / d_{i}$ and $c b_{r} a / d_{i}$ is a square, i.e., there exists $a_{0}$ with $a_{0}^{2} \in$ $\left\{b_{r} a / d_{i}, c b_{r} a / d_{i}\right\}$. Consequently,

$$
f\left(a_{0} \mathbf{w}\right)=\psi\left(a_{0}^{2} \mathbf{w} \mathbf{w}^{\top}\right)=\frac{a_{0}^{2} d_{i}}{a} \in\left\{b_{r}, b_{r} c\right\},
$$

which yields

$$
a \mathbf{w} \mathbf{w}^{\top} \in\left\{d_{i} b_{r}^{-1}\left(a_{0} \mathbf{w}\right)\left(a_{0} \mathbf{w}\right)^{\top}, d_{i} b_{r}^{-1} c^{-1}\left(a_{0} \mathbf{w}\right)\left(a_{0} \mathbf{w}\right)^{\top}\right\} \subseteq \Delta_{i, \mathbf{x}} \cup \Delta_{i, \mathbf{y}} .
$$


To continue observe that

$$
\begin{aligned}
\left|\Delta_{i, \mathbf{x}}\right| & =\alpha, \\
\left|\Delta_{i, \mathbf{y}}\right| & =\beta, \\
\left|\Delta_{\mathbf{z}}\right| & =(q-1) \gamma .
\end{aligned}
$$

We now prove that the sets $\Delta_{i, \mathbf{x}}, \Delta_{i, \mathbf{y}}, \Delta_{\mathbf{z}}(i=1, \ldots, q / p-1)$ are all disjoint. In fact, $\left.\psi\right|_{\Delta_{i, \mathbf{x}}} \equiv d_{i},\left.\psi\right|_{\Delta_{i, \mathbf{y}}} \equiv d_{i}$, and $\left.\psi\right|_{\Delta_{\mathbf{z}}} \equiv 0$, so we need only check that $\Delta_{i, \mathbf{x}} \cap \Delta_{i, \mathbf{y}}=\emptyset$. Assume otherwise, i.e., $\left(d_{i} b_{r}^{-1}\right) \cdot \mathbf{x}_{j} \mathbf{x}_{j}^{\top}=\left(d_{i} b_{r}^{-1} c^{-1}\right) \cdot \mathbf{y}_{k} \mathbf{y}_{k}^{\top}$ for some $j$ and $k$. Then $\left(d_{i} b_{r}^{-1}\right) \cdot a_{1}^{2}=\left(d_{i} b_{r}^{-1} c^{-1}\right) \cdot a_{2}^{2}$ for some nonzero $a_{1}$ and $a_{2}$ by the construction of $\mathbf{x}_{j} \mathbf{x}_{j}^{\top}$. However, this is not possible, since $c$ is non-square, so we get a contradiction.

We are now able to end the proof. We can use (19)-(21), (16)-(18), and Table 2 to deduce that

$$
\begin{aligned}
|\Delta| & =\sum_{i=1}^{q / p-1}\left|\Delta_{i, \mathbf{x}}\right|+\sum_{i=1}^{q / p-1}\left|\Delta_{i, \mathbf{y}}\right|+\left|\Delta_{\mathbf{z}}\right|=(q / p-1)(\alpha+\beta)+(q-1) \gamma \\
& \in\left\{\frac{q^{n}}{p}-1, \frac{q^{n}}{p}-1+q^{n-r / 2}\left(1-\frac{1}{p}\right), \frac{q^{n}}{p}-1-q^{n-r / 2}\left(1-\frac{1}{p}\right)\right\} .
\end{aligned}
$$

Clearly, all values can be attained for a suitable $f$.

We now prove Theorem 1.3 and Theorem 1.1.

Proof of Theorem 1.3 Suppose that $\lambda$ is an arbitrary eigenvalue and $\xi: \mathcal{S}_{n}\left(\mathbb{F}_{q}\right) \rightarrow$ $\mathbb{C} \backslash\{0\}$ is a character that satisfies (7), that is,

$$
\lambda=\sum_{M \in S} \xi(M),
$$

where $S \subseteq \mathcal{S}_{n}\left(\mathbb{F}_{q}\right)$ is the subset of all rank-one matrices. Since

$$
\xi(A)^{p}=\xi(p A)=\xi(0)=1
$$

for any $A$, there exists a map $\phi: \mathcal{S}_{n}\left(\mathbb{F}_{q}\right) \rightarrow \mathbb{F}_{p}$ such that

$$
\xi(A)=e^{2 \pi i \phi(A) / p} .
$$

The equality $\xi(A+B)=\xi(A) \xi(B)$ implies that $\phi(A+B)=\phi(A)+\phi(B)$. Hence, $\phi$ is additive, that is, linear over $\mathbb{F}_{p}$. In particular, the following two implications hold:

$$
\begin{aligned}
\phi(A)=0 & \Longrightarrow \xi(A)=1, \\
\phi(A) \neq 0 & \Longrightarrow \sum_{x \in \mathbb{F}_{p} \backslash\{0\}} \xi(x A)=\sum_{j=1}^{p-1} e^{2 \pi i j / p} .
\end{aligned}
$$


Now, let $\Delta:=\{M \in S: \phi(M)=0\}$. Since

$$
\sum_{j=1}^{p-1} e^{2 \pi i j / p}=\sum_{j=0}^{p-1} e^{2 \pi i j / p}-1=0-1=-1,
$$

we can use (22), (23), (24), and the fact that $|S|=q^{n}-1$ to deduce that

$$
\begin{aligned}
\lambda & =\sum_{M \in \Delta} \xi(M)+\sum_{M \in S \backslash \Delta} \xi(M) \\
& =|\Delta| \cdot 1+\frac{(|S|-|\Delta|) \cdot(-1)}{\left|\mathbb{F}_{p} \backslash\{0\}\right|} \\
& =|\Delta| \cdot\left(1+\frac{1}{p-1}\right)-\frac{q^{n}-1}{p-1} .
\end{aligned}
$$

The proof is complete by Lemma 3.3.

Proof of Theorem 1.1 By Theorem 2.4, $\chi\left(\Gamma_{n}\right) \geq 1+\frac{\lambda_{\max }}{-\lambda_{\min }}$. By Theorem 1.3, $\lambda_{\max }=q^{n}-1$ and $\lambda_{\min }=-q^{n-1}-1$. Since $n \geq 3$ by the assumption, it follows that $\chi\left(\Gamma_{n}\right)>q$. Let $\Gamma^{\prime}$ be a core of $\Gamma_{n}$, and let $\Phi: \Gamma_{n} \rightarrow \Gamma^{\prime}$ be any graph homomorphism. Then, we deduce that $\chi\left(\Gamma^{\prime}\right)>q$ by Lemma 2.3, which means that $\Gamma^{\prime}$ has more than $q$ vertices. By (8), the maximum clique in $\Gamma_{n}$ contains precisely $q$ vertices, so the core $\Gamma^{\prime}$ is not complete. It now follows from Lemma 3.2 that $\Gamma_{n}=\Gamma^{\prime}$ is a core. The addendum to Theorem 1.1 is now a corollary of Theorem 2.5.

We need two more lemmas to prove Theorem 1.2.

Lemma 3.4 If $q$ is odd and $c \in \mathbb{F}_{q}$ is any fixed non-square, then the set

$$
\Omega:=\left\{\left[\begin{array}{cc}
a & b \\
b & c a
\end{array}\right]: a, b \in \mathbb{F}_{q}\right\} \subseteq \mathcal{S}_{2}\left(\mathbb{F}_{q}\right)
$$

consists of $q^{2}$ pairwise nonadjacent matrices.

Proof Obviously, there are $q^{2}$ matrices in $\Omega$. To prove that they are pairwise nonadjacent, assume that $\operatorname{rk}\left(A_{1}-A_{2}\right) \leq 1$, where $A_{1}=\left[\begin{array}{ll}a_{1} & b_{1} \\ b_{1} & c a_{1}\end{array}\right]$ and $A_{2}=\left[\begin{array}{ll}a_{2} & b_{2} \\ b_{2} & c a_{2}\end{array}\right]$. Then, $\left(a_{1}-a_{2}\right)\left(c a_{1}-c a_{2}\right)=\left(b_{1}-b_{2}\right)^{2}$. If $a_{1} \neq a_{2}$, then

$$
c=\left(\frac{b_{1}-b_{2}}{a_{1}-a_{2}}\right)^{2},
$$

a contradiction. Hence, $a_{1}=a_{2}$ and consequently $b_{1}=b_{2}$, that is, $A_{1}=A_{2}$.

Recall that the trace map $\operatorname{Tr}: \mathbb{F}_{q} \rightarrow \mathbb{F}_{p}$ is surjective. If $q$ is even, then any element in $\mathbb{F}_{q}$ is a square, so there exists an nonzero $c \in \mathbb{F}_{q}$ such that $\operatorname{Tr}\left(1 / c^{2}\right)=1$. 
Lemma 3.5 If $q$ is even and $c \in \mathbb{F}_{q}$ satisfies $\operatorname{Tr}\left(1 / c^{2}\right)=1$, then the set

$$
\Omega:=\left\{\left[\begin{array}{ll}
a & b \\
b & a+c b
\end{array}\right]: a, b \in \mathbb{F}_{q}\right\} \subseteq \mathcal{S}_{2}\left(\mathbb{F}_{q}\right)
$$

consists of $q^{2}$ pairwise nonadjacent matrices.

Proof There are $q^{2}$ matrices in $\Omega$. To prove that they are pairwise nonadjacent, assume that $\operatorname{rk}\left(A_{1}-A_{2}\right) \leq 1$, where $A_{1}=\left[\begin{array}{cc}a_{1} & b_{1} \\ b_{1} & a_{1}+c b_{1}\end{array}\right]$ and $A_{2}=\left[\begin{array}{cc}a_{2} & b_{2} \\ b_{2} & a_{2}+c b_{2}\end{array}\right]$. Then, $\left(a_{1}-a_{2}\right)\left(a_{1}-a_{2}+c b_{1}-c b_{2}\right)=\left(b_{1}-b_{2}\right)^{2}$. In a field of characteristic two we deduce that

$$
x_{a}^{2}+x_{a} x_{b}+\left(1 / c^{2}\right) x_{b}^{2}=0,
$$

where $x_{a}:=a_{1}-a_{2}$ and $x_{b}:=c\left(b_{1}-b_{2}\right)$. By (6), (25) has the unique solution $x_{a}=$ $0=x_{b}$. Hence, $A_{1}=A_{2}$.

Proof of Theorem 1.2. Let $M \in \mathcal{S}_{2}\left(\mathbb{F}_{q}\right)$ be of rank one, and let $\Omega$ be as in Lemma 3.4 if $q$ is odd and as in Lemma 3.5 if $q$ is even. We claim that the family $\{x M+\Omega$ : $\left.x \in \mathbb{F}_{q}\right\}$ is a partition of $\mathcal{S}_{2}\left(\mathbb{F}_{q}\right)$. In fact, if $(x M+\Omega) \cap(y M+\Omega)$ is nonempty, then $x M+A_{1}=y M+A_{2}$ for some $A_{1}, A_{2} \in \Omega$. This is possible only if $x=y$, since $\operatorname{rk}\left(A_{1}-A_{2}\right) \neq 1$. Hence, the sets $x M+\Omega ; x \in \mathbb{F}_{q}$ are pairwise disjoint. Since $|\Omega|=q^{2}$, it follows that

$$
\left|\bigcup_{x \in \mathbb{F}_{q}}(x M+\Omega)\right|=\sum_{x \in \mathbb{F}_{q}}|x M+\Omega|=q|\Omega|=q^{3}=\left|\mathcal{S}_{2}\left(\mathbb{F}_{q}\right)\right|,
$$

so $\left\{x M+\Omega: x \in \mathbb{F}_{q}\right\}$ is truly a partition of $\mathcal{S}_{2}\left(\mathbb{F}_{q}\right)$, which means that the map $\mathcal{C}$ : $\mathcal{S}_{2}\left(\mathbb{F}_{q}\right) \rightarrow \mathbb{F}_{q}$, given by $\left.\mathcal{C}\right|_{x M+\Omega} \equiv x$, is well defined. Moreover, it is a $q$-coloring of $\Gamma_{2}$, since the two endpoints of any edge in $\Gamma_{2}$ cannot be contained in the same set $x M+\Omega$. Consequently, if $N \in \mathcal{S}_{2}\left(\mathbb{F}_{q}\right)$ is of rank one, then the map $\Phi(A)=\mathcal{C}(A) N$ is an element of $\operatorname{End}\left(\Gamma_{2}\right) \backslash \operatorname{Aut}\left(\Gamma_{2}\right)$, so $\Gamma_{2}$ is not a core. From Lemma 3.2 we infer that its core is a complete graph, that is, a maximum clique with $q$ vertices.

\section{Concluding remarks}

Remark 4.1 In matrix theory, a preserver problem demands a classification of all maps on some matrix space that leave certain subset, relation, or function invariant. In this mathematical area endomorphisms of $\Gamma_{n}$ are known as the adjacency preservers. They have an important role, since their classification can be used to classify several other preservers. In fact, if we want to classify additive preservers of certain relation, subset etc., one of the basic techniques is to first show that these maps preserve the adjacency; this procedure is nicely described in [29, Sect. 2] (see also e.g. [27, Sect. 5]). For some recent surveys on preserver problems and papers with general introduction to this subject, see e.g. [13, 24, 28]. 
Remark 4.2 It is not difficult to see that the graph $\Gamma_{2}$ does not contain an independent set with more than $q^{2}$ vertices (this follows also directly from [5, remark to Corollary 2.3]). Hence, the set $\Omega$ from Lemma 3.4/Lemma 3.5 is a maximum independent set in $\Gamma_{2}$.

Remark 4.3 Directly from the proof of Theorem 1.2 it follows that $\chi\left(\Gamma_{2}\right)=q$.

Remark 4.4 The coloring $\mathcal{C}$, and consequently the map $\Phi(A)=\mathcal{C}(A) N$, from the proof of Theorem 1.2, is additive. Namely, if $B_{1} \in x M+\Omega$ and $B_{2} \in y M+\Omega$, then $B_{1}+B_{2} \in(x+y) M+\Omega$, since $\Omega$ is closed under addition. Hence, $\mathcal{C}\left(B_{1}+B_{2}\right)=$ $\mathcal{C}\left(B_{1}\right)+\mathcal{C}\left(B_{2}\right)$. Since $\Phi \in \operatorname{End}\left(\Gamma_{2}\right)$, it follows that $\Phi$ is a rank-one preserver, that is, $\Phi(A) \neq 0$ for any $A \in \mathcal{S}_{2}\left(\mathbb{F}_{q}\right)$ of rank one.

Remark 4.5 Theorem 1.3 implies that the graph $\Gamma_{n}$ has $n+2$ distinct eigenvalues if $n$ is even and $n+1$ distinct eigenvalues if $n$ is odd. Any connected graph with diameter $d$ has at least $d+1$ distinct eigenvalues [1, Corollary 2.7]. In our case $d=n$ if $n$ or $q$ is odd, whereas $d=n+1$ if $n$ and $q$ are both even. So the number of distinct eigenvalues is minimal unless $n$ is even and $q$ is odd.

Remark 4.6 Corresponding results to Theorem 1.3 for graphs that are constructed from alternate/rectangular matrices were obtained in [7] and [6]. These graphs, as well as the graphs obtained from hermitian matrices, are distance-regular [3, Sect. 9.5]. Hence, their eigenvalues can be computed from their intersection arrays (see [3, pp. 128-129] or [1, Proposition 21.2]).

Remark 4.7 If $q$ is even, then a recursive procedure to compute the eigenvalues given by Theorem 1.3 can be deduced from [8, 32]. More precisely, in these two papers the association scheme of symmetric matrices $\operatorname{Sym}(n, q)$ and the association scheme of quadratic forms Qua $(n, q)$ are studied. In [8], recursive formulas for entries of the first eigenmatrix of Qua $(n, q)$ are obtained if $q$ is even. For example, Table A1 in [8] represents the first eigenmatrix of Qua $(2, q)$ :

$$
P=\left(\begin{array}{cccc}
1 & q^{2}-1 & \frac{q\left(q^{2}-1\right)}{2} & \frac{q(q-1)^{2}}{2} \\
1 & -1 & \frac{q(q-1)}{2} & \frac{-q(q-1)}{2} \\
1 & -1 & \frac{-q}{2} & \frac{q}{2} \\
1 & q^{2}-1 & \frac{-q(q+1)}{2} & \frac{-q(q-1)}{2}
\end{array}\right) .
$$

Since $\operatorname{Sym}(2, q)$ is formally dual to Qua $(2, q)$ [32], it follows that its first eigenmatrix equals

$$
Q=q^{3} P^{-1}=\left(\begin{array}{cccc}
1 & q^{2}-1 v & (q-1)\left(q^{2}-1\right) & q-1 \\
1 & -1 & 1-q & q-1 \\
1 & q-1 & 1-q & -1 \\
1 & -q-1 & q+1 & -1
\end{array}\right)
$$

The second column of $Q$ represents the eigenvalues of $\Gamma_{2}$. Clearly, Theorem 1.3 gives us the same values. For background on association schemes see e.g. [3, Chap. 2]. 
Acknowledgements The author is grateful to both referees and to the editor for helpful comments and suggestions.

\section{References}

1. Biggs, N.: Algebraic Graph Theory. In: Cambridge Tracts in Mathematics, No. 67. Cambridge University Press, London (1974)

2. Brouwer, A.E., Haemers, W.H.: Spectra of Graphs. Electronic book available at http://homepages. cwi.nl/ aeb/math/ipm.pdf. Accessed 12 September 2010

3. Brouwer, A.E., Cohen, A.M., Neumaier, A.: Distance-Regular Graphs. Ergeb. Math. Ihrer Grenzgeb. 3, 18 (1989)

4. Brouwer, A.E., Cohen, A.M., Neumaier, A.: Additions and Corrections to the book 'Distance-Regular Graphs'. Available at http://www.win.tue.nl/ aeb/drg/index.html. Accessed 9 April 2011

5. Cameron, P.J., Kazanidis, P.A.: Cores of symmetric graphs. J. Aust. Math. Soc. 85(2), 145-154 (2008)

6. Delsarte, P.: Bilinear forms over a finite field with applications to coding theory. J. Comb. Theory, Ser. A 25(3), 226-241 (1978)

7. Delsarte, P., Goethals, J.M.: Alternating bilinear forms over $G F(q)$. J. Comb. Theory, Ser. A 19(1), 26-50 (1975)

8. Feng, R., Wang, Y., Ma, C., Ma, J.: Eigenvalues of association schemes of quadratic forms. Discrete Math. 308(14), 3023-3047 (2008)

9. Gao, J.-F., Wan, Z.-X., Feng, R.-Q., Wang, D.-J.: Geometry of symmetric matrices and its applications III. Algebra Colloq. 3(2), 135-146 (1996)

10. Godsil, C.D.: Algebraic Combinatorics. Chapman and Hall Mathematics Series. Chapman \& Hall, New York (1993)

11. Godsil, C.D., Royle, G.F.: Algebraic Graph Theory. In: Graduate Texts in Mathematics, vol. 207. Springer, New York (2001)

12. Godsil, C.D., Royle, G.F.: Cores of geometric graphs. arXiv:0806.1300v1 (2008)

13. Guterman, A., Li, C.-K., Šemrl, P.: Some general techniques on linear preserver problems. Linear Algebra Appl. 315(1-3), 61-81 (2000)

14. Hell, P., Nešetřil, J.: Graphs and Homomorphisms. Oxford Lecture Series in Mathematics and its Applications, vol. 28. Oxford University Press, Oxford (2004)

15. Hoffman, A.J.: On eigenvalues and colorings of graphs. Graph theory and its applications. In: Proc. Advanced Sem., Math. Research Center, Univ. of Wisconsin, Madison, Wis, 1969, pp. 79-91. Academic Press, New York (1970)

16. Hua, L.-K.: Geometry of symmetric matrices over any field with characteristic other than two. Ann. Math. 50(1), 8-31 (1949)

17. Huang, L.-P.: Adjacency preserving bijection maps of Hermitian matrices over any division ring with an involution. Acta Math. Sin. Engl. Ser. 23(1), 95-102 (2007)

18. Huang, L.-P.: Geometry of $n \times n(n \geq 3)$ Hermitian matrices over any division ring with an involution and its applications. Commun. Algebra 36(6), 2410-2438 (2008)

19. Huang, W.-L.: Adjacency preserving mappings of $2 \times 2$ Hermitian matrices. Aequ. Math. 75(1-2), 51-64 (2008)

20. Huang, W.-L., Šemrl, P.: Adjacency preserving maps on hermitian matrices. Can. J. Math. 60(5), 1050-1066 (2008)

21. Huang, W.-L., Höfer, R., Wan, Z.-X.: Adjacency preserving mappings of symmetric and hermitian matrices. Aequ. Math. 67(1-2), 132-139 (2004)

22. Imrich, W., Klavžar, S.: Product Graphs, Structure and Recognition. Wiley-Interscience Series in Discrete Mathematics and Optimization. Wiley-Interscience, New York (2000)

23. Legiša, P.: Adjacency preserving mappings on real symmetric matrices. Math. Commun. (2011, in press). http://www.mathos.hr/mc

24. Li, C.-K., Pierce, S.: Linear preserver problems. Am. Math. Mon. 108(7), 591-605 (2001)

25. Lidl, R., Niederreiter, H.: Finite Fields. With a foreword by P. M. Cohn. In: Encyclopedia of Mathematics and its Applications, vol. 20. Cambridge University Press, Cambridge (1987)

26. Orel, M.: A note on adjacency preservers on hermitian matrices over finite fields. Finite Fields Appl. 15(4), 441-449 (2009)

27. Šemrl, P.: Maps on matrix spaces. Linear Algebra Appl. 413(2-3), 364-393 (2006) 
28. Šemrl, P.: Linear Preserver Problems. In: Hogben, L. (ed.), Handbook of Linear Algebra. Discrete Mathematics and its Applications. Chapman \& Hall/CRC, Boca Raton (2007). Brualdi R., Greenbaum A., Mathias R. (Associate Eds.)

29. Šemrl, P.: Geometrical techniques in linear algebra-lecture notes. In: Summer School and Advanced Workshop on Trends and Developments in Linear Algebra, Trieste (2009). Available at http://cdsagenda5.ictp.it//askArchive.php?categ=a08167\&id=a08167s126t71\&ifd=30580\&down= $1 \&$ type=lecture_notes. Accessed 12 September 2010

30. Wan, Z.-X.: Geometry of symmetric matrices and its applications II. Algebra Colloq. 1(3), 201-224 (1994)

31. Wan, Z.-X.: Geometry of Matrices: In Memory of Professor L.K. Hua (1910-1985). World Scientific, Singapore (1996)

32. Wang, Y., Wang, C., Ma, C., Ma, J.: Association schemes of quadratic forms and symmetric bilinear forms. J. Algebr. Comb. 17(2), 149-161 (2003) 